\title{
¿Psicoterapia, farmacoterapia o tratamiento combinado? Influencia de diferentes variables clínicas en la elección del tratamiento.
}

\author{
Psychotherapy, pharmacotherapy or combined treatment? Influence of different clinical \\ variables in the election of treatment.
}

\author{
Vanessa Ferreres a , Josep Pena-Garijo b, María Ballester Gil de Pareja c, Silvia Edo d, \\ Iria Sanjurjo ${ }^{\mathrm{e}}$, Luisa Ysern ${ }^{\mathrm{f}}$. \\ ${ }^{a}$ Residente de Psicología Clínica. Hospital de la Plana. Vila Real, España. ${ }^{b}$ Facultativo Especialista en \\ Psicología Clínica. Unidad Salud Mental. Hospital General de Castelló, España. Universitat Jaume I de \\ Castelló, España. ${ }^{c}$ Residente de Psicología Clínica. Hospital de la Plana. Vila Real, España. ${ }^{d}$ Facultativo \\ Especialista en Psicología Clínica. Hospital de Día. Consorcio Hospitalario Provincial de Castelló, Espa- \\ $\tilde{n} a .{ }^{e}$ Psicólogo Clínico Adjunto. Institut Pere Mata de Reus, España. ${ }^{f}$ Psicólogo Clínico Adjunto. Institut \\ Pere Mata de Reus, España.
}

Correspondencia: Josep Pena-Garijo (josepyolanda@ono.com y jpena@psb.uji.es)

Recibido: 01/07/2011; aceptado con modificaciones: 19/10/2011

RESUMEN: Introducción: Se pretenden investigar las principales variables que influyen en la elección de las diferentes intervenciones terapéuticas en una Unidad de Salud Mental pública.

Método: Estudio descriptivo sobre las historias clínicas de aquellos pacientes que iniciaron tratamiento durante el año 2008 en una USM de Castellón $(\mathrm{N}=413)$. Resultados: El tratamiento psicofarmacológico fue el más frecuentemente utilizado. La psicoterapia sola y el tratamiento combinado se utilizaron más frecuentemente en pacientes jóvenes, con estudios superiores y con trastornos de ansiedad, adaptativos o de personalidad.

Conclusiones: De los tipos de intervención estudiados, la farmacológica fue la que se aplicó con mayor probabilidad frente a la psicoterapia sola o el tratamiento combinado. La elección del tratamiento se asoció con la edad, nivel de estudios y diagnóstico. Se sospecha mayor relación con factores como la economía o la presión asistencial que con su eficacia demostrada en la literatura científica.

PALABRAS CLAVE: Psicoterapia, farmacoterapia, tratamiento combinado, salud mental.
ABSTRACT: Introduction: The present work aims to investigate the main variables that influence the election of therapeutic interventions used in a public Mental Health Unit.

Method: Descriptive study about clinical histories of those patients who initiated treatment during 2008 in a public Mental Health Unit in Castelló (Spain) ( $\mathrm{N}=413)$.

Results: Psychopharmacological treatment was the most frequently used. Psychotherapy and combined treatment were used more frequently in young patients, with superior studies and with anxiety, adaptive or personality disorders.

Conclusions: Pharmacological intervention was the treatment applied with greater probability in front of psychotherapy or combined treatment. The election of treatment was associated with age, level of studies and diagnosis. One suspects that the election of treatment is more related to economy or welfare pressure factors, than with its demonstrated effectiveness in scientific literature. KEY WORDS: Psychotherapy, pharmacotherapy, combined treatment, mental health. 
ORIGINALES Y REVISIONES

\section{Introducción}

La implantación del Modelo de Salud Mental Comunitaria, que está a la base de la Reforma Psiquiátrica, plantea como supuesto teórico una óptica integral comunitaria y la creación de equipos interdisciplinares como herramienta imprescindible para la implementación de dicho modelo.

Sin embargo, en los últimos años y en el contexto de la crisis del Sistema Sanitario Público, se observa una "regresión" hacia el Modelo Clínico Tradicional. Esto ha conducido a una perspectiva cada vez más orientada a la consulta ambulatoria y a la priorización de los tratamientos farmacológicos para el alivio rápido de los síntomas, en lugar de la inversión del tiempo que requiere la psicoterapia y las intervenciones en un contexto socio-comunitario.

Los principales factores implicados en este retroceso pueden ser tanto desviaciones que afectan a la consolidación y desarrollo del Modelo de Salud Mental Comunitario, como desviaciones que interfieran en el trabajo interdisciplinar de los equipos (1). En cuanto a las desviaciones que afectan a la consolidación y desarrollo del Modelo se destacan aspectos tales como la tendencia hacia una visión reduccionista y descontextualizada del problema, más centrada en la enfermedad como alteración orgánica del sujeto; la orientación hacia una atención individualista simplificadora en la que pierden relevancia los factores psicosociales y el abordaje interdisciplinar; la utilización de técnicas de intervención propias del Modelo Clínico Tradicional; la inclinación a establecer la remisión de la sintomatología mediante tratamientos psicofarmacológicos como único objetivo del tratamiento; la reducción del ámbito de intervención al sujeto (o sus síntomas) con desaparición progresiva de intervenciones familiares y socio-comunitarias; la desaparición progresiva de intervenciones comunitarias y preventivas y, por último, la repercusión de estas tendencias en una restricción importante de competencias a profesionales que pueden aportar modelos y estrategias de intervención de reconocida eficacia y eficiencia (1).

Por otra parte, las desviaciones que interfieren en el trabajo interdisciplinar de los equipos estarían relacionadas con las diferencias respecto a los modelos conceptuales sobre salud y enfermedad, equipo y "trabajo en equipo" entre los diferentes profesionales que lo integran; las resistencias a aceptar la complementariedad de conocimientos que aportan las otras disciplinas; las disonancias entre la formación que han recibido los profesionales y los requerimientos de nuevas técnicas y estrategias de intervención para abordar las nuevas demandas en Salud Mental; los desajustes entre los intereses y objetivos profesionales y las directrices de la administración; y, finalmente, la reducción progresiva de la capacidad de participación de los equipos en la elaboración y planificación de propuestas y actividades asistenciales (1). 
Desde que, a mediados del siglo XX, H.J. Eysenck (2) realizó la investigación que marcó el inicio del estudio de la efectividad de la psicoterapia y que afirmaba que no existen diferencias en cuanto a mejoría entre los pacientes neuróticos tratados y los no tratados, se han seguido un sin fin de investigaciones que tratan de comprobar o refutar dicha conclusión. Estudios posteriores pusieron en duda el trabajo de Eysenck, calificándolo de poco riguroso y prejuicioso y dejaron claro que la psicoterapia funcionaba (3). Ahora bien, los estudios de meta-análisis realizados también demostraban que no existían diferencias claras en los grados de mejora (tamaño del efecto) producidos por las diferentes psicoterapias predominantes de la época. Así, existe un convencimiento generalizado de que la psicoterapia es más efectiva y rápida que los cambios naturales del paciente sin tratamiento, como se concluye de las principales revisiones sobre el tema (4-8). En la actualidad, prácticamente nadie duda de la eficacia de las psicoterapias (9-13) que constituyen, junto con la farmacología o la combinación de ambas, las principales herramientas terapéuticas de los equipos de salud mental. Se han sugerido una serie de mecanismos de acción de los tratamientos combinados: los efectos positivos de la intervención conjunta se relacionan con que la medicación facilita la accesibilidad terapéutica, fomenta las funciones del Yo para la participación en psicoterapia, promueve la abreacción psicoterapéutica y además ejerce efectos positivos en expectativas, actitudes y estigma (14). Por su parte la psicoterapia facilita la adhesión al tratamiento, así como su ejercicio como coadyudante rehabilitador.

Ahora bien, no se puede especificar un único tipo de intervención, sea farmacoterapia, psicoterapia o combinada, como tratamiento idóneo para todas las enfermedades mentales. En este sentido, el objetivo de este estudio será describir y analizar la proporción de las intervenciones de tipo farmacológico, psicoterapéutico o combinado que se dan en una Unidad de Salud Mental pública, con el objetivo de determinar si se está llevando a cabo el trabajo de carácter multidisciplinario que el Modelo de Salud Mental Comunitaria plantea como abordaje de las enfermedades mentales. Se investigará la relación entre los tipos de tratamiento empleados y determinadas variables sociodemográficas y clínicas. Pretendemos con ello aportar datos que orienten acerca de estas posibles relaciones y permitan, así mismo, reflexionar sobre la adecuación de los tipos de terapias empleados a las directrices $\mathrm{u}$ orientaciones de las guías clínicas o la investigación sobre tratamientos empíricamente validados. Hipotetizamos que las relaciones encontradas no siempre irán en este sentido, por lo que es posible que existan otras influencias en la elección del tratamiento. 
ORIGINALES Y REVISIONES

\section{Material y método}

\section{Sujetos}

La muestra estuvo formada por 413 pacientes con predominio del sexo femenino y una media de edad de 43 años con desviación típica de 15. En cuanto a la edad y, con el objetivo de facilitar los análisis posteriores, se agrupó a la muestra en cuatro grandes grupos: menores de 25 años, entre 26 y 45 años, de 45 a 65 años y mayores de 66 años. El intervalo de edad más frecuente estuvo comprendido entre 26 y 45 años (47\%). El nivel de estudios primario fue el más habitual (53\%); seguido de los secundarios (correspondiente a estudios de Formación Profesional, BUP o Bachillerato), universitarios y por último la ausencia de estudios.

Respecto al diagnóstico y, por la misma razón que en el caso de la edad, también se agrupó a la muestra en seis grandes categorías diagnósticas. Por orden de frecuencia, predominaron los trastornos de ansiedad/adaptativos (40\%) y trastornos del estado de ánimo (27\%). Las restantes patologías agrupadas fueron trastornos psicóticos, trastornos de personalidad, casos de "multidiagnóstico" y una categoría residual de "otros" para todas aquellas entidades clínicas que se presentaban en una baja frecuencia y/o que no encajaban en los anteriores grupos. Dada la complejidad de los análisis a la que hubiera obligado la codificación de todos los diagnósticos, se decidió utilizar tan sólo el diagnóstico principal o el motivo principal de atención clínica, sacrificando la comorbilidad.

La tabla 1 muestra la distribución de la muestra en las diferentes variables sociodemográficas y clínicas estudiadas.

\section{Método}

Para la realización de este trabajo se llevó a cabo un estudio descriptivo sobre las historias clínicas de todos aquellos pacientes que iniciaron tratamiento durante el año 2008 en la USM 2 de Castellón que abarca parte de la zona centro de la ciudad y poblaciones del norte de la comarca de la Plana. Esta Unidad cuenta con un equipo de trabajo equilibrado: dos psiquiatras, dos psicólogos clínicos, una enfermera y una auxiliar de enfermería; condición que se consideró idónea para poder descartar posibles sesgos en los resultados debidos a la inequidad entre los profesionales en activo.

La información extraída de las historias fue de tipo sociodemográfico y clínico, concretamente, el diagnóstico clínico en el momento actual, la edad, el sexo, el nivel de estudios y el tipo de tratamiento recibido. Se limitó la elección de historias clínicas a aquellas en las que se hacía constar un diagnóstico según alguna de las clasificaciones psiquiátricas internacionales vigentes (CIE 10 o DSM IV-TR) para evitar errores en la interpretación del diagnóstico o en su agrupación posterior. Así mismo, el término "psicoterapia" se utilizó en un sentido general pero reglado, incluyendo intervenciones de diferentes escuelas, aunque predominaron la 
cognitivo-conductual, la psicodinámica y la humanista. No se incluyeron las intervenciones de tipo psicoeducativo o informativo que habitualmente se practican por enfermería o la intervención mínima que acompaña la práctica médica en general. El término "multidiagnóstico" se utilizó para incluir aquellos casos en los que no fue posible identificar un diagnóstico principal o en los que varios problemas fueron objeto de atención clínica similar.

\section{Análisis de datos}

Los datos se analizaron mediante el paquete estadístico SPSS 15.1, y se realizaron análisis descriptivos mediante tablas de contingencia y pruebas de Chicuadrado.

Tabla 1.

Distribución de la muestra

\begin{tabular}{|l|c|}
\hline Variables & $\begin{array}{c}\text { Porcentaje de casos } \\
\text { (N=413) }\end{array}$ \\
\hline Sexo & $37 \%$ \\
$\quad$ Hombres & $63 \%$ \\
Mujeres & \\
\hline Edad & $11 \%$ \\
$\quad$ entre 15-25 años & $47 \%$ \\
entre 26-45 años & $31 \%$ \\
entre 46-65 años & $11 \%$ \\
mayores 66 años & \\
\hline Nivel de estudios & $4 \%$ \\
Sin estudios & $53 \%$ \\
Primarios & $30 \%$ \\
Secundarios & $13 \%$ \\
Universitarios & \\
\hline Diagnóstico & $27 \%$ \\
Trastornos del Estado de Ánimo & $40 \%$ \\
Trastornos de Ansiedad/Adaptativos & $7 \%$ \\
Trastornos Psicóticos & $5 \%$ \\
Trastornos de la Personalidad & $14 \%$ \\
Multidiagnóstico & $8 \%$ \\
\hline Otros diagnósticos & \\
\hline
\end{tabular}

\section{Resultados}

De modo general, los resultados arrojaron diferencias significativas en cuanto los tipos de tratamientos utilizados en la Unidad de Salud Mental, siendo el tratamiento farmacológico el más frecuente $(44,8 \%)$, seguido del tratamiento combinado $(35,8 \%)$ y finalmente la psicoterapia sola $(19,4 \%)$. Nótese que el $52,2 \%$ de los pacientes recibieron algún tipo de intervención psicoterapéutica reglada. 
Los análisis también revelaron que las variables sociodemográficas y clínicas estudiadas influyeron en la elección del tipo de tratamiento para cada paciente. En concreto, respecto de la variable género, como podemos ver en la Tabla 2, no se encontraron diferencias significativas entre hombres y mujeres. En ambas muestras predominaba el tratamiento con farmacoterapia, seguido de la opción combinada y finalmente la psicoterapia sola, que fue el tratamiento menos habitual en ambos sexos (figura 1).

Tabla 2.

Distribución de tratamientos según género.

\begin{tabular}{|ll|l|c|c|c|c|}
\hline \multicolumn{2}{|l|}{ Variables } & & Psicoterapia & Fármacos & Combinado & Total \\
\hline \multirow{2}{*}{ Sexo } & \multirow{2}{*}{ Hombre } & $\mathrm{N}$ & 31 & 71 & 50 & 152 \\
& & $\%$ & $20,4 \%$ & $46,7 \%$ & $32,9 \%$ & $100,0 \%$ \\
& \multirow{2}{*}{ Mujer } & $\mathrm{N}$ & 49 & 114 & 98 & 261 \\
& & $\%$ & $18,8 \%$ & $43,7 \%$ & $37,5 \%$ & $100,0 \%$ \\
Total & $\mathrm{N}$ & 80 & 185 & 148 & 413 \\
& & & $19,4 \%$ & $44,8 \%$ & $35,8 \%$ & $100,0 \%$ \\
\hline
\end{tabular}

$\chi 2=0,90(p=0,635)$

Figura 1.

Porcentaje de tratamiento según género.

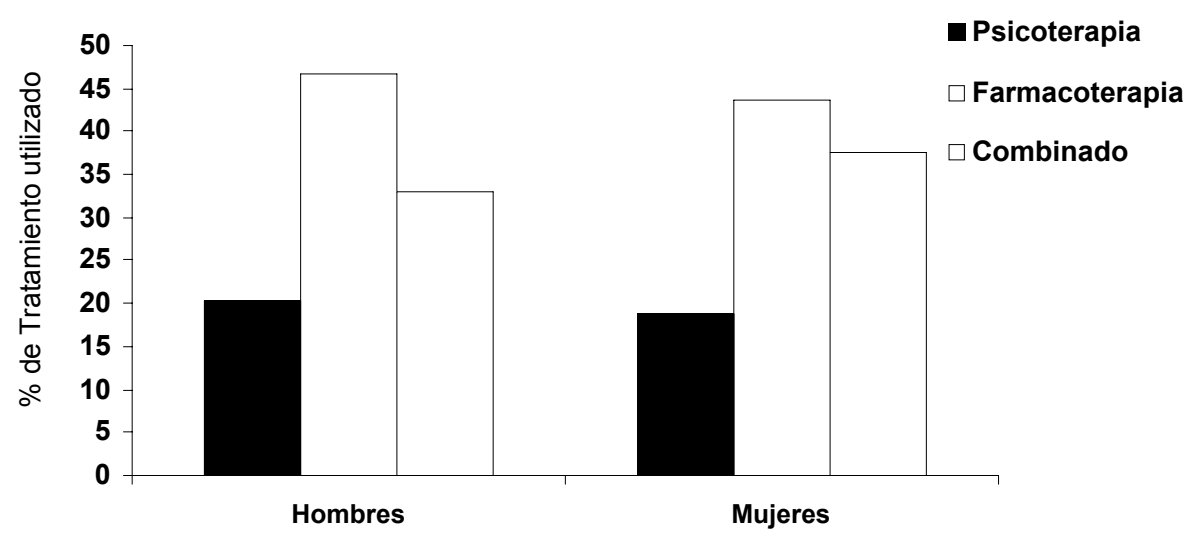

Género 
En el caso de la variable edad, como puede verse en la Tabla 3, los resultados sí reflejaron diferencias significativas en cuanto al tipo de tratamiento implementado. En los grupos de mayor edad (concretamente en los grupos de 46-65 años y en los mayores de 66 años) se llevaban a cabo con más frecuencia tratamientos farmacológicos, seguidos de tratamientos combinados y por último la psicoterapia sola. Esta tendencia (figura 2) se reducía claramente, incluso obteniéndose un patrón opuesto, a medida que se reducía la edad. Por ejemplo, en el grupo de 26-45 años el uso de la farmacoterapia disminuía y era similar al tratamiento combinado, aunque ambos tipos de intervención seguían siendo mas frecuentes, de forma significativa, que el tratamiento con psicoterapia sola. Por el contrario, en el grupo de menor edad, concretamente los adolescentes, el uso de los fármacos se redujo notablemente y fue el tratamiento combinado junto con la psicoterapia los que aparecieron con mayor frecuencia aunque, en este caso, no se obtuvieron diferencias significativas al comparar los tres tipos de tratamiento.

Tabla 3.

Distribución de tratamientos según edad.

\begin{tabular}{|c|c|c|c|c|c|c|}
\hline \multicolumn{2}{|c|}{ Variables } & & Psicoterapia & Farmacoterapia & Combinado & Total \\
\hline \multirow[t]{2}{*}{ Sex } & \multirow[t]{2}{*}{$15-25$ años } & $\mathrm{N}$ & 17 & 10 & 20 & 47 \\
\hline & & $\%$ & $36,2 \%$ & $21,3 \%$ & $42,6 \%$ & $100,0 \%$ \\
\hline \multirow{2}{*}{\multicolumn{2}{|c|}{ 26-45 años }} & $\mathrm{N}$ & 46 & 74 & 73 & 193 \\
\hline & & $\%$ & $23,8 \%$ & $38,3 \%$ & $37,8 \%$ & $100,0 \%$ \\
\hline \multirow{2}{*}{\multicolumn{2}{|c|}{ 46-65 años }} & $\mathrm{N}$ & 16 & 67 & 43 & 126 \\
\hline & & $\%$ & $12,7 \%$ & $53,2 \%$ & $34,1 \%$ & $100,0 \%$ \\
\hline \multirow{2}{*}{\multicolumn{2}{|c|}{$>66$ años }} & $\mathrm{N}$ & 1 & 34 & 12 & 47 \\
\hline & & $\%$ & $2,1 \%$ & $72,3 \%$ & $25,5 \%$ & $100,0 \%$ \\
\hline \multirow{2}{*}{\multicolumn{2}{|c|}{ Total }} & $\mathrm{N}$ & 80 & 185 & 148 & 413 \\
\hline & & $\%$ & $19,4 \%$ & $44,8 \%$ & $35,8 \%$ & $100,0 \%$ \\
\hline
\end{tabular}

$\chi 2=38,78(p=0,000)$

Figura 2.

Porcentaje de tratamiento según edad.

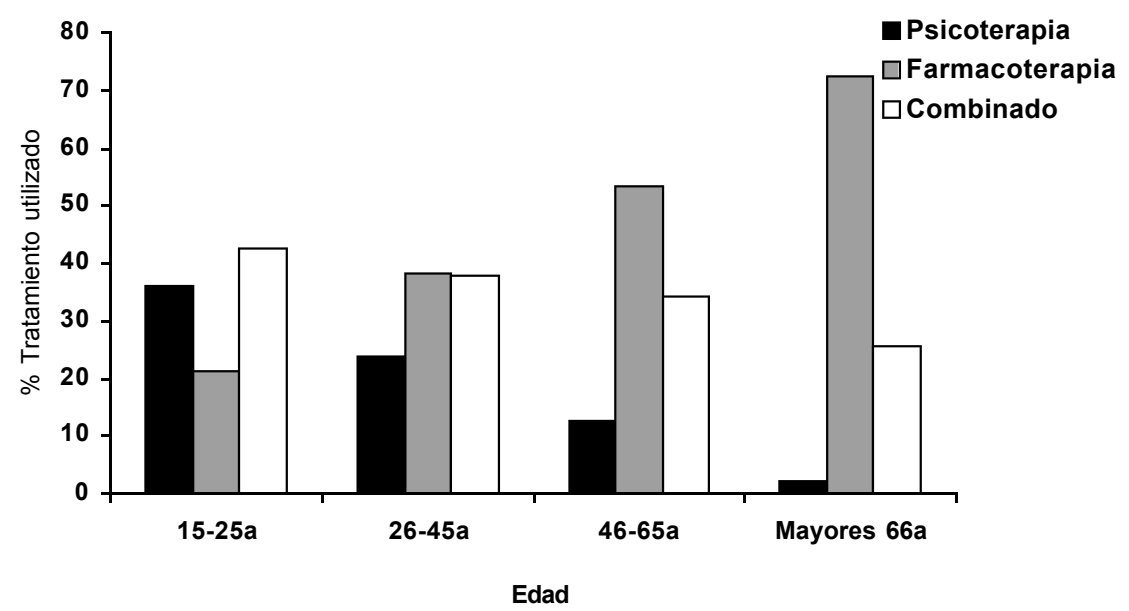


También se obtuvieron diferencias significativas en el tipo de tratamiento según el nivel de estudios (Tabla 4), de manera que el uso de intervenciones psicoterapéuticas solas era más frecuente en los pacientes con un mayor nivel de estudios, frente a la farmacoterapia, que seguía el patrón opuesto y era claramente más utilizada en los pacientes sin estudios (donde prácticamente era el único tipo de tratamiento utilizado) y de menor formación (estudios primarios y secundarios). En el caso del tratamiento combinado, la tendencia fue mucho menos evidente, presentando una mayor aplicación que el resto de tratamientos en el caso de los pacientes universitarios y algo menor que la farmacoterapia en niveles de instrucción primarios y secundarios. De cualquier forma, la farmacoterapia fue el tratamiento mas utilizado en todos los casos excepto en el caso de los universitarios, entre los que destacaban las intervenciones de tipo combinado (figura 3).

Tabla 4.

Distribución de tratamientos según nivel de estudios.

\begin{tabular}{|c|c|c|c|c|c|}
\hline Variables & & Psicoterapia & Farmacoterapia & Combinado & Total \\
\hline \multirow[t]{2}{*}{ Estudios Primarios } & $\mathrm{N}$ & 35 & 107 & 79 & 221 \\
\hline & $\%$ & $15,8 \%$ & $48,4 \%$ & $35,7 \%$ & $100,0 \%$ \\
\hline \multirow[t]{2}{*}{ Secundarios } & $\mathrm{N}$ & 29 & 51 & 39 & 119 \\
\hline & $\%$ & $24,4 \%$ & $42,9 \%$ & $32,8 \%$ & $100,0 \%$ \\
\hline \multirow[t]{2}{*}{ Superiores } & $\mathrm{N}$ & 15 & 13 & 28 & 56 \\
\hline & $\%$ & $26,8 \%$ & $23,2 \%$ & $50,0 \%$ & $100,0 \%$ \\
\hline \multirow[t]{2}{*}{ Sin estudios } & $\mathrm{N}$ & 1 & 14 & 1 & 16 \\
\hline & $\%$ & $6,3 \%$ & $87,5 \%$ & $6,3 \%$ & $100,0 \%$ \\
\hline \multirow[t]{2}{*}{ No consta } & $\mathrm{N}$ & 0 & 0 & 1 & 1 \\
\hline & $\%$ &, $0 \%$ &, $0 \%$ & $100,0 \%$ & $100,0 \%$ \\
\hline \multirow[t]{2}{*}{ Total } & $\mathrm{N}$ & 80 & 185 & 148 & 413 \\
\hline & $\%$ & $19,4 \%$ & $44,8 \%$ & $35,8 \%$ & $100,0 \%$ \\
\hline
\end{tabular}

$\chi^{2}=28,20(p=0,000)$

Figura 3.

Porcentaje de tratamiento según nivel de estudios.

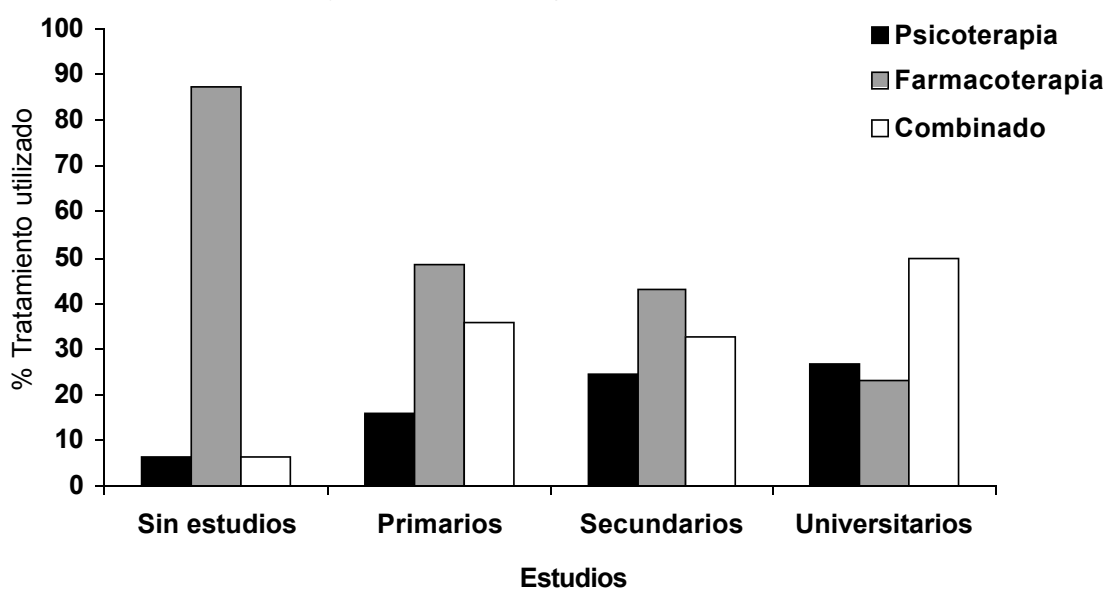


El tipo de diagnóstico también resultó ser una variable determinante en cuanto al tipo de tratamiento utilizado. Concretamente, según se aprecia en la Tabla 5 , se observó que la farmacoterapia fue significativamente la más utilizada en los trastornos psicóticos y en los trastornos del estado de ánimo. La psicoterapia se utilizó con más frecuencia que la farmacoterapia en los trastornos de ansiedad/ adaptativos, aunque menos que la terapia combinada. En los Trastornos de Personalidad el uso de la psicoterapia y del tratamiento farmacológico fue similar, pero mayor en ambos casos que la terapia combinada. La combinación de los dos tipos de tratamiento se dio con más frecuencia en los casos de Multidiagnóstico y en los Trastornos de Ansiedad y Adaptativos aunque, en estos últimos, este tipo de intervención no difería de forma significativa del uso de la psicoterapia sola (figura 4).

Tabla 5.

Distribución de tratamientos según diagnóstico.

\begin{tabular}{|c|c|c|c|c|c|}
\hline Variables & & Psicoterapia & Fármacos & Combinado & Total \\
\hline \multirow[t]{2}{*}{ Primarios } & $\mathrm{N}$ & 0 & 23 & 4 & 27 \\
\hline & $\%$ & $0 \%$ & $85,2 \%$ & $14,8 \%$ & $100,0 \%$ \\
\hline \multirow[t]{2}{*}{ Estado Ánimo } & $\mathrm{N}$ & 10 & 73 & 30 & 113 \\
\hline & $\%$ & $8,8 \%$ & $64,6 \%$ & $26,5 \%$ & $100,0 \%$ \\
\hline Ansiedad & $\mathrm{N}$ & 50 & 44 & 70 & 164 \\
\hline$\stackrel{\mathscr{c}}{=}$ & $\%$ & $30,5 \%$ & $26,8 \%$ & $42,7 \%$ & $100,0 \%$ \\
\hline 茎 Personalidad & $\mathrm{N}$ & 8 & 9 & 3 & 20 \\
\hline & $\%$ & $40,0 \%$ & $45,0 \%$ & $15,0 \%$ & $100,0 \%$ \\
\hline \multirow[t]{2}{*}{ Multi-diagnóstico } & $\mathrm{N}$ & 3 & 24 & 31 & 58 \\
\hline & $\%$ & $5,2 \%$ & $41,4 \%$ & $53,4 \%$ & $100,0 \%$ \\
\hline \multirow[t]{2}{*}{ Otros } & $\mathrm{N}$ & 9 & 12 & 10 & 31 \\
\hline & $\%$ & $29,0 \%$ & $38,7 \%$ & $32,3 \%$ & $100,0 \%$ \\
\hline \multirow[t]{2}{*}{ Total } & $\mathrm{N}$ & 80 & 185 & 148 & 413 \\
\hline & $\%$ & $19,4 \%$ & $44,8 \%$ & $35,8 \%$ & $100,0 \%$ \\
\hline
\end{tabular}

$\chi 2=81,78(p=0,000)$

Figura 4.

Porcentaje de tratamiento según diagnóstico.

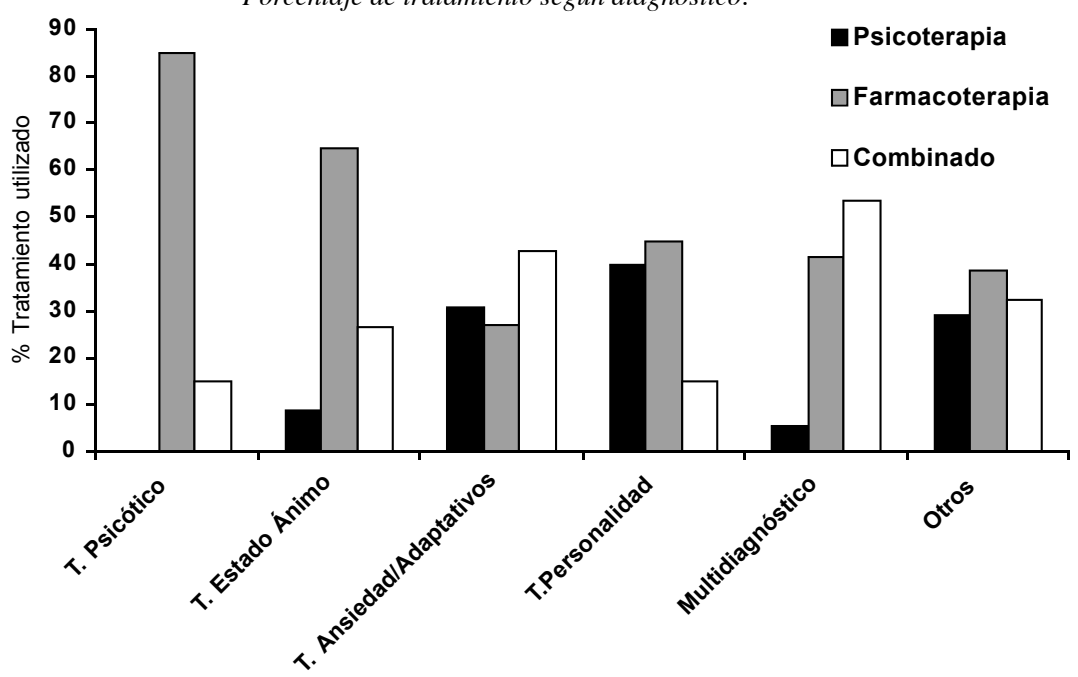

Diagnóstico 


\section{Discusión}

Los resultados del estudio concuerdan con lo esperado teniendo en cuenta el estado de "regresión" del Modelo de Salud Comunitaria al que nos referíamos en la introducción. De los tres tipos de intervención estudiados, la farmacológica fue la que se aplicó con mayor probabilidad en la Unidad de Salud Mental sobre la que se realizó el presente trabajo. Sin embargo, la selección de un tipo de tratamiento u otro pareció estar relacionada con determinadas variables de carácter tanto clínico, como socio-demográfico tales como la edad, el nivel de estudios y el tipo de diagnóstico, aunque no con otras como el género. Respecto a la psicoterapia, resultó ser el tipo de intervención más frecuentemente utilizada en pacientes jóvenes y con mayores niveles de instrucción; frente a la farmacoterapia, que fue el tratamiento más habitual en pacientes mayores y con menos estudios. El tratamiento combinado fue otra importante opción de tratamiento, principalmente en el caso de los pacientes con estudios superiores, independientemente de la edad.

Como también era de esperar, la orientación diagnóstica de los pacientes fue otra importante razón para escoger un tipo de intervención u otra, observándose una preferencia por las intervenciones farmacológicas en los trastornos psicóticos y los afectivos frente a los tratamientos psicoterapéuticos. Estos últimos fueron una opción frecuente en los Trastornos de Personalidad, de Ansiedad y en los Trastornos Adaptativos. Sin embargo, en ningún caso la psicoterapia sola fue la opción más habitual. En los casos de Multidiagnóstico, la opción del tratamiento combinado resultó ser la más frecuentemente elegida.

Estos datos no siempre se asemejan a los ofrecidos por la literatura científica respecto a la eficacia de los tratamientos (15). Llaman la atención algunas cuestiones tales como el poco uso de los tratamientos combinados en el caso de los trastornos psicóticos cuando, hoy por hoy, se plantea como tratamiento necesario la combinación de fármacos antipsicóticos y terapias psicosociales y rehabilitadoras. En este sentido, con la introducción de los antipsicóticos atípicos se ha favorecido este tipo de abordaje. Estos fármacos han potenciado el rol de los esfuerzos rehabilitadores psicosociales, porque sostienen la promesa de la recuperación a largo plazo más que la paliación de síntomas que permitían las previas generaciones de antipsicóticos (16). La interpretación de este hallazgo puede justificarse por la presencia de otros dispositivos en la red asistencial de la zona (como por ejemplo los CRIS o los Hospitales de Día), dispositivos donde se llevan a cabo las intervenciones de carácter más psicosocial y rehabilitador, de lo que se puede deducir que estas intervenciones se dejan en manos de los profesionales que prestan sus servicios en dichos dispositivos. Otra posible explicación estaría relacionada con el escaso tiempo que llevaban en tratamiento estos pacientes en el momento de la recogida de datos (no más de un año) y que explicaría un primer abordaje de tipo 
farmacológico. En cualquier caso, se ha demostrado la eficacia de las intervenciones psicológicas en las psicosis (17) tanto en la rehabilitación como en la intervención en los primeros episodios (18).

En cuanto a los trastornos afectivos, también se observó un escaso uso de la psicoterapia frente al tratamiento farmacológico y, a diferencia de lo que se describe en la literatura (19), el tratamiento combinado no resultó ser el abordaje más habitual. Un posible sesgo en este sentido podría tener que ver con la no distinción de la gravedad del trastorno afectivo al seleccionar los grupos, aspecto que se ha visto muy relacionado a la hora de elegir un tipo de intervención u otro; ni la diferenciación entre trastornos depresivos y bipolares. De cualquier forma, es obvio el escaso uso de la psicoterapia, aún cuando se han demostrado ampliamente sus ventajas frente a la farmacoterapia en una gran variedad de situaciones clínicas. Recientes revisiones de la literatura apuntan en este sentido (20,21). La bibliografía arroja resultados similares tanto para los tratamientos farmacológicos como psicológicos. El Instituto Nacional de Salud Mental (NIMH) de Estados Unidos realizó en 1977 una ambiciosa investigación con el objetivo de comparar terapia cognitiva, psicoterapia interpersonal y psicofármacos en pacientes diagnosticados de depresión mayor. Los resultados de esta investigación informaron de beneficios diversos de las intervenciones: Los psicofármacos actuaban más rápidamente mientras que las psicoterapias evitaban mejor las recaídas en periodo de seguimiento. En cuanto al tratamiento combinado en depresión, parece existir consenso al afirmar que la combinación psicoterapia-psicofármacos está especialmente indicada en pacientes con depresión severa (22-24). Ahora bien, la combinación no ha mostrado ser más efectiva en casos leves o moderados (25) o encuentran que la terapia combinada es más eficaz que la psicoterapia sola (26). Sin embargo, en la práctica clínica el tratamiento combinado resulta ser el más habitual, ya que los estudios realizados hasta el momento plantean esta intervención como más efectiva que la farmacoterapia o la psicoterapia sola, especialmente en los casos de depresión más severa o crónica $(19,27)$.

Otro dato a tener en cuenta fue el importante porcentaje de intervenciones combinadas, semejante a la psicoterapia sola, en el caso de los Trastornos de Ansiedad. La literatura científica refleja claramente el uso de los tratamientos psicológicos, principalmente de orientación cognitivo conductual, para este tipo de trastornos (15). Se han publicado diversos estudios que plantean la idoneidad de unos u otros tipos de intervenciones $(28,29)$. Muchos trastornos de ansiedad pueden ser mejor tratados con intervenciones de orientación cognitivo-conductual que con las farmacológicas. Los tratamientos cognitivo-conductuales para trastornos fóbicos y otros trastornos de ansiedad suelen producir, frecuentemente, efectos más duraderos que los tratamientos farmacológicos y, además, no conllevan los riesgos de dependencia que acompañan al uso de algunas intervenciones farmacológicas en estos trastornos, como es 
el caso de las benzodiacepinas (15). Para el trastorno de pánico, existe evidencia de las ventajas del tratamiento combinado, pero las diferencias encontradas respecto a la monoterapia frecuentemente no son significativas (30). En cuanto a la fobia social también hay cierta evidencia de las ventajas del tratamiento combinado (31) y recientemente se han publicado estudios que demuestran la superioridad del tratamiento combinado respecto a la monoterapia (32). También existen datos que informan de las ventajas del tratamiento combinado para el trastorno obsesivo-compulsivo (33$35)$ y el trastorno de ansiedad generalizada $(36,37)$.

En el caso de los Trastornos de Personalidad (TP), hubo un incremento importante de los tratamientos psicológicos solos que no difirió de la elección de la farmacoterapia sola y que, curiosamente, superaron a los tratamientos combinados. La literatura actual describe intervenciones psicológicas eficaces en los TP, como la Terapia dialéctico conductual para el Trastorno Límite $(38,39)$ u otras intervenciones tanto dinámicas, conductuales o integradas en los TP en general (40), así como la eficacia de tratamientos farmacológicos en el Trastorno Límite (41).

Finalmente, existe una creciente evidencia de la eficacia de las terapias cognitivo-conductuales llamadas de "tercera generación" en la prevención de recaídas en la depresión mayor recidivante (42) y en la reducción sintomática en grupos de pacientes transdiagnósticos (43), intervenciones en formato de grupo y de duración breve que, indudablemente, repercutirían en una mejor relación coste-beneficio.

Sin embargo, la realidad es que son muchos los pacientes que, tratados con fármacos debido a la mayor rapidez de este tipo de intervención y a la sobrecarga asistencial, acaban por no beneficiarse de la intervención psicológica. Además, en muchos casos, los pacientes pueden ya venir medicados por su Médico de Atención Primaria cuando son remitidos a la Unidad de Salud Mental, aspecto que no se contempló en la recogida de datos y que podría estar produciendo un aparente incremento en el porcentaje de tratamientos combinados encontrados. Ocurrió otro posible sesgo con los Trastornos Adaptativos, entre los que no se diferenció si existía un predominio de sintomatología depresiva o ansiosa. De cualquier forma, a un porcentaje importante de estos pacientes que, inicialmente, reciben ambos tipos de tratamiento por su clínica más aguda, a lo largo de la intervención, se les terminó retirando el fármaco (o lo abandonaron) y acabaron siendo tratados exclusivamente con psicoterapia.

El estudio presenta algunas otras limitaciones, algunas de ellas inherentes a los estudios descriptivos. Además, se observan otros problemas metodológicos, como la escasa finura de los diagnósticos, el hecho de que la muestra proviene tan solo de una Unidad de Salud Mental concreta, la indefinición del tipo de psicoterapia aplicada, los abandonos, los seguimientos a medio plazo o la eficacia real de los tipos de intervenciones estudiadas. Estos aspectos deberían tenerse en cuenta en futuras investigaciones. 
En cualquier caso, parece que el mayor uso de intervenciones farmacológicas podría estar relacionado con el aparente mayor coste de las intervenciones psicológicas (sobre todo de tiempo), con la menor dotación de psicoterapeutas entrenados en alguna forma de psicoterapia, con la industria farmacéutica y con la presión asistencial, factores que inciden en que se acabe eligiendo con mayor frecuencia la opción farmacoterapéutica. El proyecto IAPT, llevado a cabo en Gran Bretaña, revela la conveniencia de mejorar el acceso a las terapias psicológicas en Atención Primaria y demuestra la eficacia, eficiencia y efectividad de este tipo de tratamientos (8).

Parece que queda un largo camino por recorrer para que en nuestros Servicios Públicos se instaure un Modelo de Salud Mental en el cual se conceda la importancia adecuada a los tratamientos más eficaces sin priorizar, como se está haciendo, el uso de la farmacoterapia. Los resultados del presente estudio sugieren que existe una relación entre variables como la edad, el nivel de estudios y el tipo de diagnóstico con la elección del tratamiento. Sin embargo, frente a las recomendaciones de las guías clínicas y los resultados de la literatura especializada sobre terapias validadas empíricamente, los datos que encontramos muestran ciertas discrepancias. Sospechamos que existen otros factores que influyen en la elección de la terapia (presión asistencial, economía, políticas sanitarias, industria farmacéutica, falta de formación de los profesionales en prácticas basadas en la evidencia, etc.). Los datos no permiten concluir más allá pero abren la puerta a otros estudios en los que cabría investigar estas supuestas influencias externas a la clínica y al desarrollo de buenas prácticas e instar a nuestros gestores a tomar las medidas necesarias para conseguir el objetivo de una mejor asistencia, así como facilitar el acceso a los mejores tratamientos a la mayor cantidad de población posible.

\section{BIBLIOGRAFÍA:}

(1) Pallarés T y Vallejo F. Psicología Clínica en los Servicios Comunitarios. Asociación Española de Neuropsiquiatría. Cuadernos Técnicos 1997; 2:25-32.

(2) Eysenck HJ. The effects of psychotherapy: An evaluation. J Consult Psychol 1952;16:319324

(3) Sloane RB, Staples FR, Cristol AH, Yorkston N y Whipple K. Psychotherapy versus behaviour therapy. Cambridge MA: Harvard Univ. Press, 1975.

(4) American Psychiatric Associaton. Psycho-therapy research: Methodological and efficacy issues: Washington DC, 1982.

(5) Lambert MJ y Bergin AE. Achievements and limitations of psychotherapy research. En: Freedheim DK, editor. History of psychotherapy: A century of change. Washington, DC: American Psychological Association, 1992; p, 360-390.

(6) Lamber, MJ, Shapiro DA y Bergin AE. The effectiveness of psychotherapy. En: Garfield SL y Bergin AE editors. Handbook of psychotherapy and behavior change. 3d ed. Nueva York: Wiley. 1986. 
(7) Luborsky L, Singer B y Luborsky L. Comparative studies of psychotherapies: Is it true that "Everyone has won and all must have prizes"? Arch Gen Psychiatry 1975;32: 995-1008.

(8) Turpin G, Richards D, Hope R y Duffy R. Mejorando el acceso a las terapias psicológicas: una iniciativa nacional para asegurar la prestación de terapias de alta calidad basadas en la evidencia. Papeles del Psicólogo 2008;29(3): 271-280.

(9) Echeburua E. ¿Qué terapias psicológicas son eficaces? Un reto ante el año 2000. Revista de Psicopatología y Psicología Clínica 1998;3: 149-160.

(10) Chambless DL y Ollendick TH. Empirically Supported Psychological Interventions: Controversies and Evidence. Annu Rev Psychol 2001;52: 685-716.

(11) Bados A, García Brau E. y Fusté A. Eficacia y utilidad clínica de la terapia psicológica. Int J Clin Health Psicol. 2002; 2(3): 477-502.

(12) Echeburúa E. Retos de futuro de la psicología clínica. Análisis y Modificación de Conducta 2008; 34: 209-207.

(13) Echeburua E. Del Corral P y Salaberria K. Efectividad de las terapias psicológicas: un análisis de la realidad actual. Revista de Psicopatología y Psicología Clínica 2010;15(2): 85-99.

(14) Klermar GL, Weissman MM, Markowitz JC, Glick I, Wilner PJ, Mason B et al. Medication and psychotherapy. En: Bergin AE, Garfield SL, editors. Handbook of psychotherapy and behavior change. 4th ed. New York: Wiley and Sons, 1994; p, 734-782.

(15) Sammons M y Schmidt N. Tratamientos combinados de los trastornos mentales. Guia de intervenciones psicologicas y farmacologicas. Bilbao: DDB, 2004.

(16) Spaulding W, Johnson D y Coursey R. Treatment and rehabilitation of schizophrenia. En: Sammons M y Schmidt N, editors. Combined Treatments for Mental Disorders: A guide to psychological and pharmacological interventions. Washington DC: American Psychological Association, 2001; p, 161-190.

(17) García I. Evolución de la psicoterapia en la esquizofrenia. Rev. Asoc. Esp. Neuropsiq 2008; 101: 9-25.

(18) Vallina $\mathrm{O}$ y Lemos S. Tratamientos psicológicos eficaces para la esquizofrenia. Psichotema 2001;13(3):345-364.

(19) Pettit JW, Voelz ZR, Joiner TM. Combined Treatments for depression. En: Sammons M y Schmidt N, editors. Combined Treatments for Mental Disorders: A guide to psychological and pharmacological interventions. Washington DC: American Psychological Association, 2001; p,131-159.

(20) Kriston L, Von Wolff A, Hölzel L. Effectiveness of psychotherapeutic, pharmacological, and combined treatments for chronic depression: A systematic review. BMC Psychiatry 2010;10: 95.

(21) Cuijpers P, van Strraten A, Hollon SD y Andersson G. The contribution of active medication to combined treatments of psychotherapy and pharmacotherapy for adult depression: A meta-analysis. Acta Psychiatr Scand 2010;121(6): 415-423.

(22) Blackburn IN, Bishop S, Glen AIM, Whalley L J y Christie JE. The efficacy of cognitive therapy in depression: a treatment trial using cognitive therapy and pharmacotherapy, each alone and in combination. Br J Psychiatry 1981; 139: 181-9.

(23) Chaudhry HR, Najam N y Naqvi A. The value of amineptine in depressed patients treated with cognitive behavioural psychotherapy. Hum Psychopharmacol 1998;13: 419-23.

(24) Grambal A, Sigmundova Z, Hunková M y PraПło J. Comparison of the effect of pharmacotherapy and the combination of psychotherapy and pharmacotherapy in the treatment of depressive disorder. Eur Psychiatry 2011; 26(1): 632. 
(25) Wexler BE, Cicchetti DV. The outpatient treatment of depression: implications of outcome research for clinical practice. J Nerv Ment Dis 1992;180: 277-86.

(26) Cuijpers P, van Straten P, Warmerdam, L y Gerhard Andersson G. Psychotherapy versus the combination of psychotherapy and pharmacotherapy in the treatment of depression: a meta-analysis. Depress Anxiety 2009;26: 279-288.

(27) Oestergaard S, Moldrup O. Optimal duration of combined psychotherapy and pharmacotherapy for patients with moderate and severe depression: A meta-analysis. J Affect Disord 2011;131: 24-36.

(28) Zwanzger P, Diemer J, Jabs B. Comparison of combined psycho and pharmacotherapy with monotherapy in anxiety disorders: controversial viewpoints and clinical perspectives. J Neural Transm 2009; 116(6):759-65.

(29) Otto MW, Behar E, Smits JAJ y Hofmann S. Combining pharmacological and cognitive behavioral therapy in the treatment of anxiety disorders. En: Antony M y Stein M, editors. Oxford handbook of anxiety and related disorders. New York: Oxford University Press, 2009; p, 429-440.

(30) Foa EB, Franklin ME y Moser J. Context in the clinic: How well do cognitive-behavioral therapies and medications work in combination? Biol Psychiatry 2001;10: 987-997.

(31) Blomhoff S, Haug TT, Hellström K, Holme I, Humble M, Madsbu HP, et al. Randomised controlled general practice trial of sertraline, exposure therapy and combined treatment in generalized social phobia. Br J Psychiatry 2001;179: 23-30.

(32) Blanco C, Heimberg RG, Schneier FR, Fresco DM, Chen H, Turk CL, et al. A placebocontrolled trial of phenelzine, cognitive behavioral group therapy, and their combination for social anxiety disorder. Arch Gen Psychiatry 2010;67(3): 286-295.

(33) Cottraux J, Mollard E, Bouvard M, Marks I, Sluys M, Nury AM, et al. A controlled study of fluvoxamine and exposure in obsessive-compulsive disorder. Int Clin Psychopharmacol 1990;5:17-30.

(34) Hohagen F, Winkelmann G, Räsche-Rauschle H, Hand I, König A, Münchau N, et al. Combination of behaviour therapy with fluvoxamine in comparison with behaviour therapy and placebo: Results of a multicentre study. Br J Psychiatry 1998;35(Suppl.): 71-78.

(35) Simpson HB, Foa E. Liebowitz MR, Ledley DR, Huppert J, Cahill S et al. A Randomized, Controlled Trial of Cognitive-Behavioral Therapy for Augmenting Pharmacotherapy in Obsessive-Compulsive Disorder. Am J Psychiatry 2008;165:621-630.

(36) Power KG, Simpson R.J, Swanson V, y Wallace LA. Controlled comparison of pharmacological and psychological treatment of generalized anxiety disorder in primary care. Br J Gen Pract 1990;40(336): 289-294.

(37) Siev J, Chambless DL. Specificity of treatment effects: cognitive therapy and relaxation for generalized anxiety and panic disorders. J Consult Clin Psicol. 2007;75(4):513-22.

(38) Linehan M, editor. Skills training manual for treating borderline personality disorder. Nueva York: Guilford Press, 1993

(39) Harned MS, Chapman AL, Dexter-Mazza ET, Murray A, Comtois KA, Linehan MM. Treating co-occurring Axis I disorders in recurrently suicidal women with borderline personality disorder: a 2-year randomized trial of dialectical behavior therapy versus community treatment by experts. J Consult Clin Psychol. 2008;76(6):1068-75.

(40) Misiego JM, Rubio I, Santos JM, Carrasco JL, Pérez A, Molina R, et al. Psicoterapia de los Trastornos de la Personalidad. Actas Españolas de Psiquiatría 2004;32(1):37-52.

(41) Lieb K, Völlm B, Rücker G, Timmer A y Stoffers JM. Pharmacotherapy for borderline personality disorder: Cochrane systematic review of randomised trials. Br J Psychiatry 2010;196:4-12. 
(42) Miró T, Perestelo-Pérez L, Pérez J, Rivero A, González M, de la Fuente J y Serrano P. Eficacia de los tratamientos psicológicos basados en mindfulness para los trastornos de ansiedad y depresión: Una revisión sistemática. Revista de Psicopatología y Psicología Clínica 2011;16(1): 1-16.

(43) Pena-Garijo J, Ferreira M y Carmona Y. Aplicación de un programa de Mindfulness en un grupo de pacientes con trastornos mentales comunes. Acta Psiquiatr Psicol Am Lat 2011;57(2): 122-130. 